\title{
Colistín en la era post-antibiótica
}

\author{
Alejandro Aguayo, Sergio Mella, Gisela Riedel, Helia Bello, Mariana Domínguez y Gerardo González-Rocha
}

\section{Colistin in the post-antibiotic era}

One of the most important features of the post-antibiotic era in the late $20^{\text {th }}$ century is the resurgence of colistin for the treatment of extensively drug resistant gram-negative bacteria (XDR). Colistin is a narrow spectrum antibiotic, active against microorganisms with clinical significance such as Acinetobacter baumannii, Pseudomonas aeruginosa and Klebsiella pneumoniae. Nowadays its toxicity is lower, partly explained by better pharmaceuticals and management of the critically ill patients. However, there has been much confusion regarding the dosage of the drug, its name and labeling, therefore, experts have recommended using a common language about this polymyxin. The lack of PK/PD studies for colistin is perhaps the main weakness of this area of knowledge, even though the before mentioned approach has contributed with new ways to manage and calculate the dose of this antimicrobial. Indeed, the efficiency of colistin in association with a second agent in reducing mortality has not been demonstrated.

Key words: Colistin, polymyxin, post-antibiotic era, bacterial drug-resistance, structure-activity relationship, combination therapy.

Palabras clave: Colistín, polimixina, era post-antibiótica, resistencia bacteriana, relación estructura-actividad, terapia combinada.

\section{Introducción}

L a reintroducción de colistín como terapia de última línea para el tratamiento de infecciones producidas por bacilos gramnegativos (BGN) extensamente resistentes a fármacos (XDR, extensively drug resistant), es decir, resistentes a carbapenémicos y otros agentes antibacterianos y sólo susceptibles a colistín y tigeciclina, es a nuestro juicio la característica más importante de la era post-antimicrobiana ${ }^{1-8}$.

Colistín fue introducido en la práctica clínica en la década de los 509,10; sin embargo, su uso se discontinuó en forma sistemática con el desarrollo de otros agentes activos sobre BGN y que, en general, se asociaban a menores fenómenos de toxicidad ${ }^{9-11}$. Sin embargo, el incremento sostenido e insospechado de la resistencia antimicrobiana, particularmente en BGN, y la ausencia del desarrollo de nuevos fármacos activos sobre este grupo en los últimos 30 años, motivó la reintroducción sistemática de esta polimixina ${ }^{10-11}$. Así, actualmente su uso se ha incrementado en forma notable concomitantemente a la descripción de mejor tolerancia a la descrita en décadas previas, pero también a la creciente descripción de aislados resistentes ${ }^{11-14}$.

Se revisará a continuación la estructura química y elementos básicos de relación estructura-actividad del complejo de las polimixinas. Posteriormente, se analizará la actividad antimicrobiana de dicha familia, características farmacocinéticas y farmacodinámicas y los principales mecanismos de resistencia descritos a la fecha.

\section{Estructura química}

De acuerdo a Bryskier ${ }^{15,16}$, las polimixinas son lipopéptidos cíclicos, caracterizados por una cadena peptídica que se encuentra unida a un ácido graso, y pueden ser divididos en lineales y cíclicos (Figura 1).

\section{Lipopéptidos cíclicos}

Incluyen un amplio grupo de moléculas, algunas con uso clínico, como las polimixinas y la daptomicina, las que son estructuralmente similares, pero con espectro de acción diferente. De acuerdo a su actividad biológica se distinguen: actividad sobre bacterias gramnegativas; actividad sobre bacterias grampositivas, agentes antifúngicos $\mathrm{y}$ agentes antimicobacterianos.

\section{Lipopéptidos activos sobre bacterias gramnegativas}

La molécula principal del grupo es el complejo de las polimixinas, que corresponde a un decapéptido unido a una cadena de ácidos grasos. Este decapéptido contiene un loop cíclico de siete aminoácidos entre el grupo amino de la cadena lateral del ácido di-amino-butírico en posición 4 y el grupo carboxilo del carbono terminal del residuo 10 de treonina. Este loop se une a través de una cadena de tres aminoácidos al ácido graso amino terminal ${ }^{11,15,17}$ (Figura 2). Otras características químicas incluyen: residuos de ácido alfa-gamma-di-amino-butírico, lo cual hace que esta molécula a pH 7,4 sea policatiónica. Asimismo, la presencia de la cadena lateral de ácidos grasos y los sus- 
tituyentes en posiciones 6 y 7 explican la hidrofobicidad de la molécula ${ }^{15,17}$. Al igual que muchos otros péptidos antimicrobianos esta mezcla de grupos hidro y lipofílicos hacen de las polimixinas moléculas anfipáticas, propiedad físico-química que es esencial para el mecanismo de acción de este antimicrobiano ${ }^{17}$.

Diferentes tipos de polimixinas han sido caracterizadas desde el año 1947 como producto de fermentación de Paenibacillus polymyxa. Cada grupo es definido por una letra (desde A a T) de acuerdo a los residuos de aminoácidos presentes en su secuencia, especialmente en base a la diferencia de los aminoácidos en posición 6 y 7. A su vez, cada uno de éstos han sido sub-clasificados según el ácido graso presente en el grupo amino terminal ${ }^{17}$ (Tabla 1). De los diferentes grupos de polimixinas identificados hasta ahora sólo las polimixinas B y E tienen uso clínico ${ }^{17}$. Dado que en nuestro medio sólo se dispone para uso sistémico de polimixina E, denominado colistín, nos enfocaremos en el análisis de este sub-grupo.

La formulación farmacéutica de colistín es la molécula inactiva colistimetato de sodio (CMS), también denominado colistín metanosulfonato, e incluye a las polimixinas E1 y E2 como sus dos mayores componentes ${ }^{18}$. Debe recordarse que antimicrobianos de uso tan habitual como gentamicina también incluyen distintos tipos de variedades de la molécula activa ${ }^{19}$. Además, colistín es generalmente definido por la presencia de una D-leucina en posición 6 y una L-leucina en posición 7 (Figura 2). Los residuos de aminoácidos son de configuración levógira, excepto la D-leucina en posición 6. Por otra parte, tal como se mencionó previamente, el ácido graso en la posición amino terminal, que contiene en forma variable entre siete a nueve átomos de carbono, permite la sub-

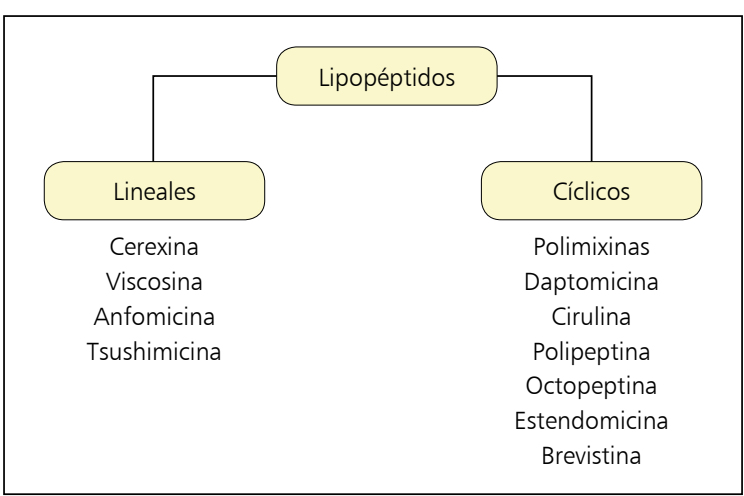

Figura 1. Clasificación de los lipopéptidos. Peptide antibiotics. Bryskier A (1ed).

clasificación del grupo de la polimixina E (E1, E2, E3, E4, E7 y E8-Ile).

\section{Mecanismo de acción}

Las bacterias gramnegativas poseen una estructura especial, que por lo demás es una barrera de paso para muchas substancias -entre ellas los antimicrobianos- nos referimos a la membrana externa ${ }^{20}$. Esta membrana posee una capa interna, de naturaleza fosfolipídica y una externa, el lipopolisacárido (LPS).

Estructuralmente, el LPS de la bacteria gramnegativa está compuesto por tres dominios: el antígeno $O$, un polímero de oligosacáridos; el núcleo o core, un oligosacárido; el lípido A o endotoxina que funciona como ancla del LPS a la membrana externa ${ }^{21}$. El lípido A corresponde a un disacárido de glucosamina unido por un enlace B-1' 6 , esterificado en cuatro posiciones con ácidos grasos saturados y fosforilado en los extremos 1' y $4^{\prime 22}$ (Figura 3).

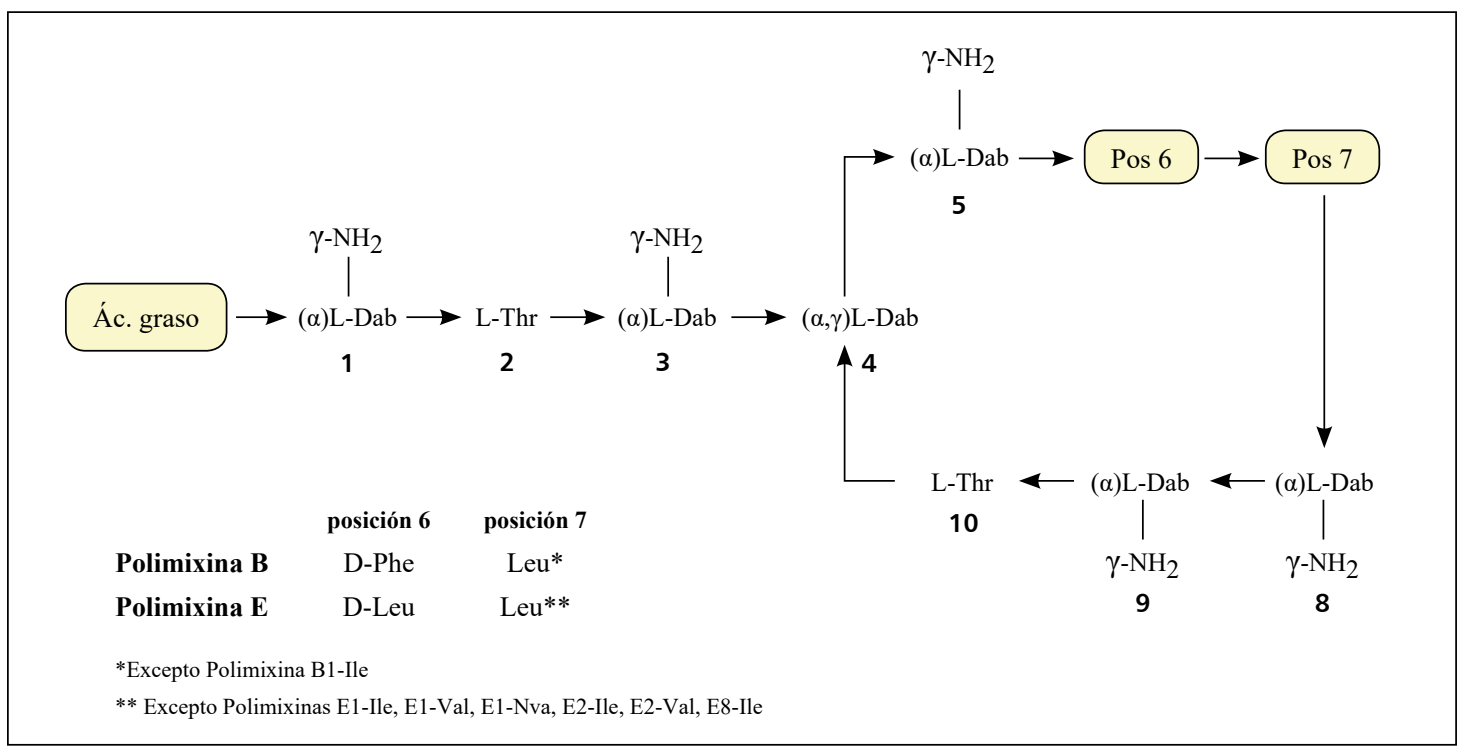

Figura 2. Estructura básica de las polimixinas. L: levógiro; D: dextrógiro; Dab: ácido diaminobutírico; Thr: treonina; Phe: fenilalanina; Leu: leucina; lle: isoleucina; Val: valina; Nva: norvalina. Se aprecia el loop cíclico de siete aminoácidos (posición 4-10) unidos a través de una cadena de tres aminoácidos (posición 1-3) a un ácido graso. Además, los diferentes residuos en posición 6 y 7 determinan las distintas polimixinas. Por último, el ácido graso determina el subtipo (Lancet Infect Dis 2006; 6: 589-601). 
Tabla 1. Clasificación de las polimixinas en distintos tipos (según el aminoácido en posición 6 y 7) y subtipos (según el ácido graso terminal)

\section{Tipos y subtipos de polimixinas}

Polimixina

B1

B1-lle

B2

$\mathrm{B} 3$

B4

B5

B6

E1

E2

E3

E4

E7

E1-Ile

E1-Val

E1-Nva

E2-Ile

E2-Val

E8-Ile

Ácido graso

(S)-6-metiloctanoil

(S)-6-metiloctanoil

Aminoácido 6

D-Phe

D-Phe

D-Phe

D-Phe

Octanoil

Heptanoil

D-Phe

D-Phe

3-hidroxi-6-metiloctanoil

D-Phe

D-Leu

D-Leu

D-Leu

D-Leu

D-Leu

D-Leu

(S)-6-metiloctanoil

(S)-6-metiloctanoil

(S)-6-metiloctanoil

6-metilheptanoil

6-metilheptanoil

7-metilnonanoil

D-Leu

D-Leu

D-Leu

D-Leu

D-Leu
Aminoácido 7

Leu

Ile

Leu

Leu

Leu

Leu

Leu

Leu

Leu

Leu

Leu

Leu

Ile

val

Nva

Ile

val

Ile

D: dextrógiro; Phe: fenilalanina; Leu: leucina; Ile: isoleucina; Val: valina; Nva: norvalina.

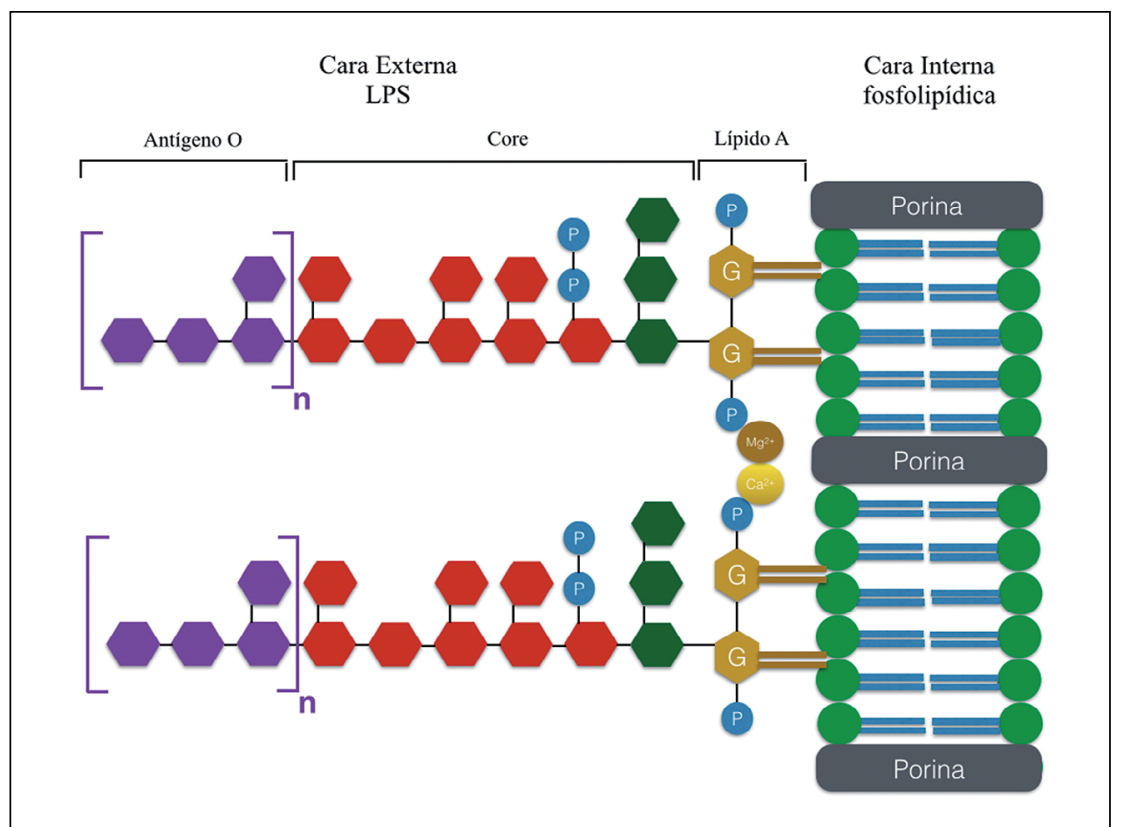

Figura 3. Estructura de la membrana externa. El LPS constituye la cara externa, conformado por el antígeno $\mathrm{O}$, el núcleo o core y el lípido A; la cara interna es de naturaleza fosfolipídica. El lípido A es un disacárido de glucosamina unido por un enlace $\beta-1$ ' -6 , esterificado en cuatro posiciones con ácidos grasos y fosforilado en los extremos 1' y 4'. En relación a los fosfoésteres del lípido A, los cationes divalentes $\mathrm{Ca}^{2+}$ y $\mathrm{Mg}^{2+}$ unen y estabilizan las moléculas de LPS. LPS: lipopolisacárido; G: glucosamina; P: fosfato; $\mathrm{Mg}^{2+}$ : ion magnesio; $\mathrm{Ca}^{2+}$ : ion calcio.
Para entender el mecanismo de acción de colistín es necesario saber que existen dos cationes divalentes, $\mathrm{Mg}^{2+}$ $\mathrm{y} \mathrm{Ca}^{2+}$ asociados a los fosfoésteres del lípido A, los cuales tienen un rol estructural muy relevante al tener la función de unir y estabilizar las moléculas de LPS adyacentes ${ }^{23}$ (Figura 3). De esta forma, la membrana externa de la bacteria gramnegativa se configura como una barrera no sólo mecánica, sino también electrostática, con una elevada carga aniónica repulsiva, conferida por las fracciones fosfoéster del lípido A, así como también por los fosfatos y carboxilatos de los azúcares de las otras dos estructuras del LPS (core y antígeno $\mathrm{O}$ ).

El sitio blanco de las polimixinas es el lípido A del LPS y para su acción es crucial la naturaleza anfipática de estos antimicrobianos. Como se mencionó anteriormente, las polimixinas a $\mathrm{pH}$ fisiológico $(7,4)$ configuran una molécula policatiónica, al cargarse positivamente las aminas presentes en los residuos de ácido di-amino-butírico (en posición 1, 3, 5, 8 y 9). De esta forma se genera, inicialmente, una atracción electrostática entre la molécula de polimixina y los fosfatos del lípido A cargados negativamente. Luego de esta interacción inicial se produce un desplazamiento de los cationes bivalentes, $\mathrm{Ca}^{2+} \mathrm{y} \mathrm{Mg}^{2+}$, que como ya se mencionó, estabilizan el LPS bacteriano (Figura 4a). Una vez desestructurado el LPS, se inserta la molécula antimicrobiana en la membrana externa a través de sus segmentos hidrófobos, es decir, mediante la cola de ácido graso de la posición amino-terminal y a través de los residuos hidrófobos de la posición 6 y 7 del antimicrobiano (Figura 4a). Después de insertada la molécula de polimixina condiciona un deterioro del ensamblaje de los ácidos grasos de la molécula del lípido A, provocando una expansión estructural de la membrana externa. Posteriormente, y a través de mecanismos no bien dilucidados, se genera una fusión de la capa interna de la membrana externa (la que se encuentra orientada hacia el espacio periplásmico) con la cara externa de la membrana citoplasmática. Se cree que esto genera cambios en su estructura fosfolipídica, lo que finalmente determina que se pierda la resistencia osmótica de la bacteria, que lleva al efecto bactericida ${ }^{24}$ (Figura $4 b$ ).

\section{Actividad antimicrobiana}

En general, el espectro clínico de todas las polimixinas es similar. Sólo existen algunas pequeñas diferencias cuantitativas de su actividad in vitro. El espectro de colistín incluye a la mayoría de las bacterias gramnegativas, excepto los géneros Neisseria, Proteus, Providencia, Brucella, Serratia y Edwardsiella ${ }^{1025}$. Además, no es activo frente a Burkholderia mallei y B. cepacia. Las especies no cubiertas con mayor importancia clínica quedan representadas en el acrónimo PPBS (Proteus- 
Providencia-Burkholderia-Serratia). Colistín tampoco tiene actividad sobre bacterias grampositivas, ni anaerobios. Por lo anterior, las polimixinas son consideradas antimicrobianos de reducido espectro.

La importancia del uso de colistín radica en su acción frente a patógenos gramnegativos XDR, incluyendo Pseudomonas aeruginosa, Acinetobacter baumannii, Klebsiella pneumoniae y Stenotrophomonas maltophi${ }_{\text {lia }}{ }^{26}$. Según el CLSI la susceptibilidad para P. aeruginosa y $A$. baumannii es definida con una $\mathrm{CIM} \leq 2 \mathrm{mg} / \mathrm{L}$, mientras que el EUCAST coincide con su contraparte estadounidense en el punto de corte para A. baumannii, pero establece una CIM $\leq 4 \mathrm{mg} / \mathrm{L}$ para $P$. aeruginos $a^{27,28}$.

\section{Farmacocinética-farmacodinámica (PK/PD)}

La introducción de colistín en la década de 1950 se llevó a cabo en una época en que los estudios de farmacocinética cumplían estándares de calidad menores y, por lo tanto, el proceso de aceptación de un fármaco era menos riguroso que el actual. Su retirada por toxicidad (a inicios de 1980) determinó que existiera una falta de interés por este antimicrobiano y, por lo tanto, el conocimiento de esta polimixina se detuvo. Esto determinó un gran problema al momento de reintroducir el colistín en la práctica clínica como tratamiento de última línea frente a BGN, ya que no existía información PK/PD que guiara apropiadamente su uso ${ }^{29}$. Desde su reintroducción, ha existido información contradictoria respecto a la eficacia y seguridad de este antimicrobiano. Uno de los principales hechos que generan confusión es que la misma molécula tenga distintos preparados comerciales, con etiquetados diferentes y dosis recomendadas incongruentes. Así, en Reino Unido (y Europa) se usa Colomycin ${ }^{\circledR}$ y en Estados Unidos de América Coly-Mycin $\mathrm{M}^{\circledR}$ Parenteral. Como se puede comprobar, el problema ya se inicia sólo con lo similar de la denominación comercial del preparado. Más confusión produce el hecho que el mismo producto venga etiquetado de distintas formas: "colistimetato de sodio" en el caso de Colomycin y "actividad de colistín base" (CBA) para Coly-Mycin M Parenteral. Se suma a lo anterior, el hecho que la etiqueta de cada frasco viene en diferentes unidades de medida: Unidades Internacionales (UI) para Colomycin y miligramos de CBA para el caso de Coly-Mycin M parenteral ${ }^{30,31}$. En relación a lo anterior las equivalencias son las siguientes: 1 millón de $\mathrm{UI}=80$ $\mathrm{mg}$ de $\mathrm{CMS}=30 \mathrm{mg}$ de $\mathrm{CBA}^{32}$.

Para no dejar dudas respecto al desorden que se ha visto con el uso de colistín, mencionaremos el hecho que la dosis recomendada por los distintos laboratorios para sus respectivos preparados (que insistimos, es el mismo producto) son inconsistentes entre $\mathrm{si}^{33,34}$, tal como queda resumido en la Tabla 2. Lo anterior ha generado que, incluso en publicaciones de las más importantes revistas

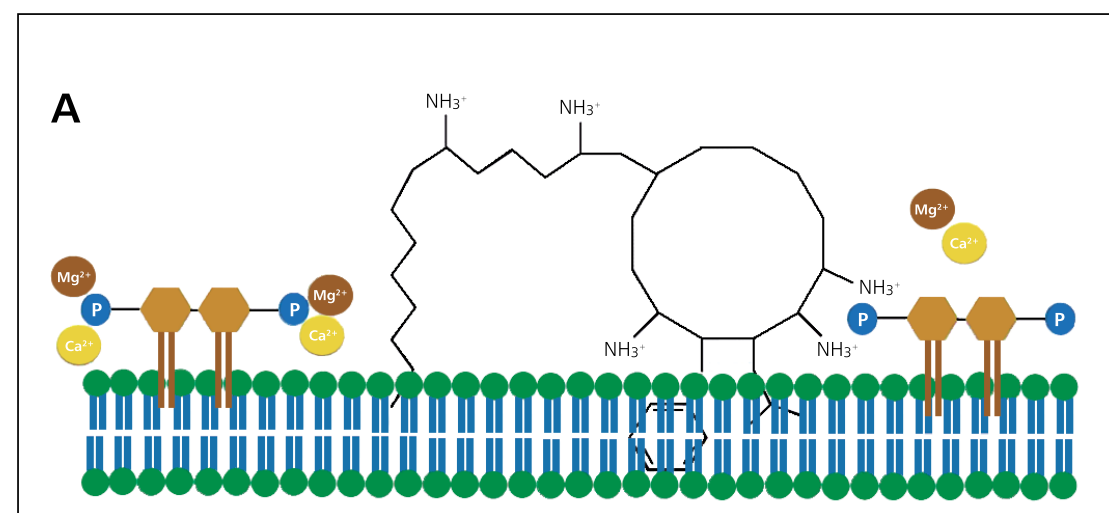

B

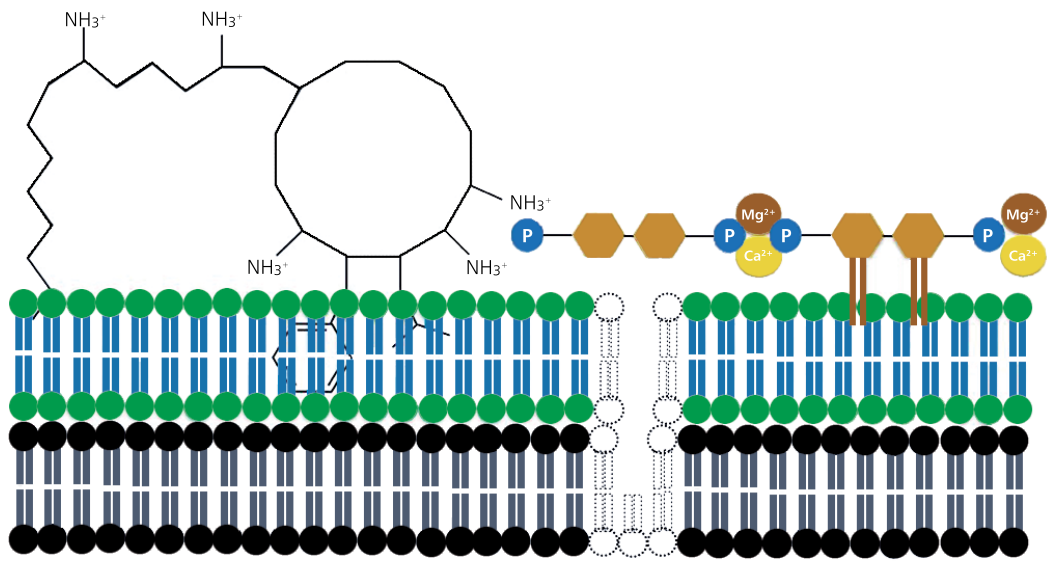

Figura 4. Mecanismo de acción de las polimixinas. A: Inicialmente se genera una atracción electrostática entre la polimixina, de carga neta positiva, y el lípido A, de carga negativa, lo que genera el desplazamiento repulsivo de los cationes divalentes que estabilizan el LPS, a lo que sigue la inserción del antimicrobiano a través de sus residuos hidrófobos en la membrana externa; B: Una vez inserta la polimixina, se genera una alteración estructural de la membrana citoplasmática que llevaría a la lisis bacteriana por pérdida de la resistencia osmótica. LPS: lipopolisacárido; G: glucosamina; P: fosfato; $\mathrm{Mg}^{2+}$ : ion magnesio; $\mathrm{Ca}^{2+}$ : ion calcio. Intencionalmente no se esquematizó el core ni el antígeno O.

de enfermedades infecciosas a nivel mundial, se hayan producido errores respecto a la denominación y dosificación de polimixina $\mathrm{E}^{35}$. Por lo mismo, en los últimos años se ha hecho un llamado a la comunidad médica internacional para unificar criterios de dosificación y para evitar denominaciones que sólo generan confusión; por ejemplo, equivalencias en Unidades Internacionales de $\mathrm{CBA}^{33,36}$.

Muchos de los problemas en el conocimiento de la farmacocinética de colistín provienen del hecho que se administra en su forma inactiva, la prodroga $\mathrm{CMS}^{37}$. Este último es un compuesto inestable que es capaz de convertirse en colistín, tanto in vivo como en fluidos ex vivo. Por lo mismo, si la prodroga no es rápida ni adecuadamente almacenada para su estudio, se puede seguir 


\begin{tabular}{|c|c|c|}
\hline & Colomycin & Coly-Mycin M Parenteral \\
\hline Principales distribuidores & Reino Unido & $\begin{array}{l}\text { Estados Unidos } \\
\text { Australia }\end{array}$ \\
\hline Etiquetado por frasco & $500.000,1.000 .000$ o $2.000 .000 \mathrm{UI}$ & 150 mg de actividad de colistín base \\
\hline Dosis recomendada & $\begin{array}{l}\leq 60 \mathrm{~kg}: 50.000 \mathrm{Ul}-75.000 \mathrm{UI} / \mathrm{kg} / \mathrm{día} \\
>60 \mathrm{~kg}: 1-2 \text { millones UI } 3 \text { veces al día }\end{array}$ & 2,5-5,0 mg CBA/kg/día \\
\hline $\begin{array}{l}\text { Dosis máxima recomendada por el fabricante para paciente de } 60 \mathrm{~kg} \\
\text { (calculado como } \mathrm{mg} \text { de CMS) }\end{array}$ & $360 \mathrm{mg}$ CMS & $800 \mathrm{mg}$ CMS \\
\hline
\end{tabular}

hidrolizando en colistín, incluso después de obtenida una muestra y, por lo tanto, generar niveles falsamente elevados del compuesto activo ${ }^{29}$. Otro problema de los estudios antiguos de farmacocinética fue que por mucho tiempo no se pudo diferenciar el CMS del colistín en las pruebas de concentraciones plasmáticas. Sólo a partir del año 2000 se comenzó a usar nuevos métodos cromatográficos (HPLC; LC-MS/MS) capaces de diferenciar cuantitativamente ambos compuestos ${ }^{38}$. Por lo mismo los datos farmacocinéticos previos al año 2003 pueden considerarse como no válidos ${ }^{29}$.

El primer estudio farmacocinético moderno, que significó un gran avance en el conocimiento de colistín, fue el realizado por Plachouras y cols. ${ }^{39}$, en un grupo de 18 pacientes críticos. Un hallazgo relevante fue que las concentraciones plasmáticas previstas tras la primera dosis de CMS (en un esquema de 3 millones de UI cada $8 \mathrm{~h}$ iv) serían $0,6 \mathrm{mg} / \mathrm{L}$ y en el estado de equilibrio $2,3 \mathrm{mg} / \mathrm{L}$. Esto es extremadamente importante, considerando que el límite propuesto para considerar a los microorganismos susceptibles a colistín es $2 \mathrm{mg} / \mathrm{L}$ y, si la concentración del antimicrobiano es insuficiente (como se vio tras la primera dosis), habrá un impacto en la morbi-mortalidad y en emergencia de resistencia. Por lo mismo, los autores sugirieron una carga de hasta 9 millones de UI de CMS al inicio del tratamiento y, además, se cuestionó por primera vez el punto de corte de susceptibilidad para colistín, ya que con el esquema de dosificación habitual, a menudo se obtendrán concentraciones plasmáticas sub-inhibitorias de colistín. Otro hallazgo importante de este estudio es el lento incremento de la concentración plasmática del antimicrobiano, demorándose (sin dosis de carga) aproximadamente $60 \mathrm{~h}$ en llegar al estado de equilibrio. Y por último, la hidrólisis de CMS en colistín en pacientes críticos fue lenta, demorándose aproximadamente siete horas en llegar a la concentración plasmática máxima post dosis.
El segundo estudio farmacocinético moderno relevante fue el realizado por Garonzik y cols ${ }^{40}$, también llevado a cabo en un grupo pacientes críticos. Además de confirmar la necesidad de una dosis de carga, ayudó a comprender mejor la farmacocinética de colistín en diversos espectros de función renal. Los autores revelaron que la concentración plasmática de colistín se eleva en insuficiencia renal, presumiblemente al no eliminarse CMS, que queda más disponible para hidrolizarse posteriormente en el fármaco activo. Lo anterior hace necesario su ajuste en casos de deterioro de la función renal. Además, confirmaron que CMS/colistín son eficientemente aclarados por hemodiafiltración veno-venosa y durante hemodiálisis. Los autores proponen la primera fórmula para calcular la dosis de carga (basada en el peso corporal) y de mantención (que incorpora el aclaramiento de creatinina), además de hacer recomendaciones para pacientes en hemodiálisis intermitente y en terapias continuas de sustitución renal. Los autores, además, sugieren que la administración de CMS sea en tres dosis en pacientes con función renal normal, lo que se asociaría a menor toxicidad en modelos animales ${ }^{41}$, y a menor emergencia de resistencia en estudios in vitro ${ }^{42}$.

Si aceptamos la recomendación de Garonzik y cols ${ }^{40}$, el objetivo plasmático de colistín a alcanzar es $2,5 \mathrm{mg} / \mathrm{L}$, algo así como un punto intermedio entre eficacia y toxicidad. Sin embargo, es necesario mencionar que con esta concentración sérica del antimicrobiano el fAUC 0-24 es aproximadamente $24 \mu \mathrm{gh} / \mathrm{mL}$ lo que en estudios in vitro se ha asociado, en el mejor de los casos, a actividad bacteriostática contra BGN con CIM entre 0,5-2,0 mg/ $\mathrm{L}^{43,44}$. Desafortunadamente, no se cuenta con estudios que evalúen el impacto en eficacia y seguridad de mayores dosis del antimicrobiano. Lo anterior es la base teórica para que muchos expertos recomienden terapias antimicrobianas combinadas.

La penetración de colistín a través de la barrera hemato-encefálica es pobre, y se estima aproximadamente 
en $5 \%{ }^{45}$, lo que aumentaría hasta un rango de $25-67 \%$ durante una meningitis. Su penetración también es baja en el tracto biliar, líquido pleural y $\operatorname{articular}^{10}$.

Colistín produce una rápida muerte bacteriana dependiente de la concentración, con un insignificante efecto post-antibiótico ${ }^{46,47}$. Recientes modelos in vitro y en animales, proponen que la relación del área bajo la curva concentración plasmática libre versus tiempo en relación a la CIM (fAUC/CIM) es el parámetro PK/PD que mejor se relaciona con la actividad y eficacia antibacteriana del colistín contra $P$. aeruginosa y A. baumannii ${ }^{43,44}$. Actualmente hay estudios en marcha, que buscan profundizar los conocimientos en farmacocinética de CMS/colistín con el objetivo de optimizar la forma de usar nuestro "último recurso terapéutico".

\section{Mecanismos de resistencia}

La resistencia a colistín, si bien ha sido descrita, es poco frecuente ${ }^{48}$. Esto se relaciona muy probablemente con el poco uso que se le dio por muchos años, cuando fue retirado por toxicidad. No obstante, y como era de esperar, a medida que va aumentando su uso se van describiendo diferentes mecanismos de resistencia, algunos de ellos sorprendentes (Figura 5). El principal mecanismo es a través de modificación del sitio blanco del antimicrobiano, en este caso del LPS, específicamente del lípido A. Al momento de la redacción de este artículo, el mecanismo global más importante de resistencia está mediado por mutaciones en los genes del sistema regulatorio de PhoPQ-PmrAB, tanto en MgrB (regulador negativo de PhoQ) como en la quinasa PmrB, que finalmente producen un aumento en la transcripción de complejos-arn, permitiendo la biosíntesis de moléculas catiónicas que cambian la carga neta del LPS y, por tanto, disminuyen la unión de las polimixinas ${ }^{49}$. Así, Adams y cols ${ }^{50}$ describieron el sistema PmrAB (polimyxin resistance), describiendo que la mutación o el incremento de expresión de los genes $p m r \mathrm{~A}$ y $p m r \mathrm{~B}$ es seguido de un aumento en la cantidad de fosfoetanolamina y/o 4-amino-4-desoxi-1-arabinosa (LAra4N) en el lípido A. La adición covalente de estas moléculas, que son de carga positiva, hacen que la carga neta del lípido A sea menos negativa, lo que altera el primer mecanismo de unión de la polimixina con su sitio blanco, que es la atracción electrostática ${ }^{51}$. Todo lo anterior se traduce en disminución de la afinidad del antimicrobiano por su sitio blanco ${ }^{52}$. Otro mecanismo asociado al aumento de resistencia a polimixinas es la adición de cadenas adicionales de ácidos grasos a la estructura del lípido A, lo que haría a la membrana externa menos penetrable para las polimixinas ${ }^{53}$.

La capacidad adaptativa de una bacteria para lograr ventajas evolutivas, le permite dejar de expresar estruc-

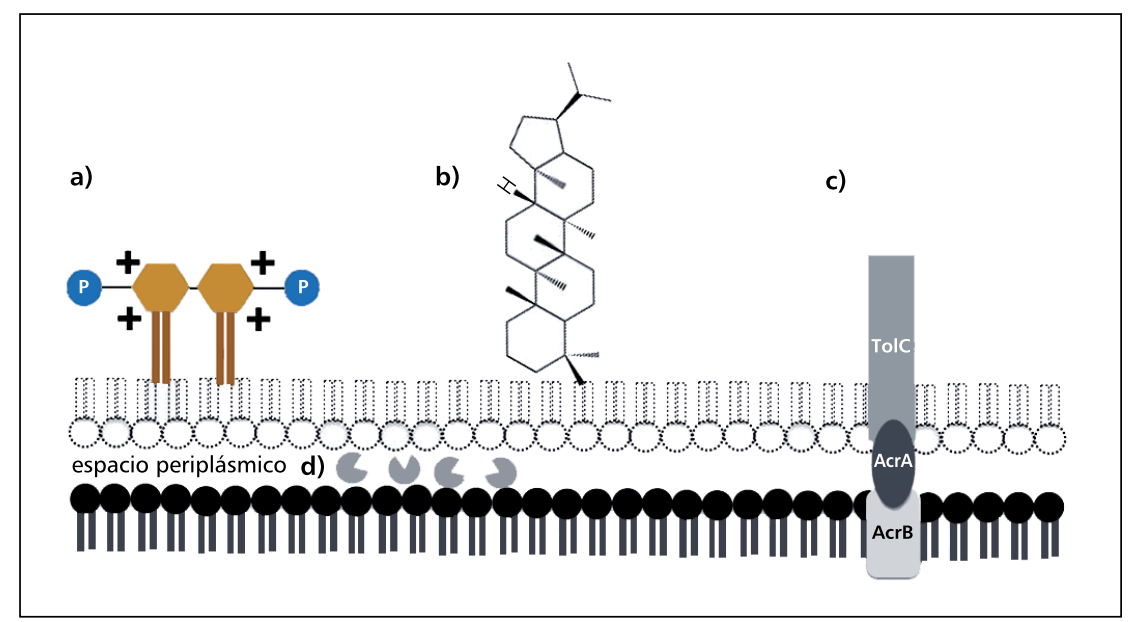

Figura 5. Mecanismos de resistencia a las polimixinas. A) la mutación del sistema PmrAB lleva a una disminución de la carga neta negativa del lípido A, alterando la interacción electrostática inicial de la polimixina con su sitio blanco; B) Los hopanoides se han asociado a la insusceptibilidad de algunas cepas a colistín; C) Bombas de eflujo capaces de movilizar las polimixinas; D) Proteasas del espacio periplásmico capaces de hidrolizar colistín. P: fosfato. Intencionalmente no se esquematizó el core ni el antígeno $\mathrm{O}$.

turas determinantes. Es así como se ha descrito en $A$. baumannii la pérdida completa de la producción de LPS ${ }^{54}$. Diferentes eventos moleculares, tales como deleciones, inserciones o mutaciones puntuales pueden inactivar cualquiera de los tres principales genes de la vía sintética del lípido A: $l p x A, l p x C$, y $l p x D^{55,56}$. La ausencia del sitio blanco hace inviable la acción de las polimixinas. La bacteria compensa la pérdida del LPS aumentando la síntesis de otras estructuras, tales como fosfolípidos, lipoproteínas y poli- $\beta-1,6-N$-acetilglucosamina ${ }^{56}$.

Existen otros mecanismos de resistencia no relacionados a la modificación del LPS. En $K$. pneumoniae se ha relacionado resistencia a polimixinas con la cantidad de polisacáridos capsulares ${ }^{57}$. Además, se ha visto que la expresión de ciertas proteínas de la membrana citoplasmática, como bombas de expulsión, se asocia a resistencia en bacterias gramnegativas. En $P$. aeruginosa se ha descrito resistencia a colistín por sobre-expresión del sistema de bombas de expulsión mexAB-oprM ${ }^{58}$. Así mismo, en $K$. pneumoniae y Escherichia coli se ha asociado resistencia a polimixinas y la presencia de la bomba de expulsión AcrAB-TolC y en Burkholderia vietnamiensis la bomba multidroga NorM ${ }^{59-61}$.

Los hopanoides son compuestos pentacíclicos presentes en algunas especies bacterianas que presentan una similitud estructural con los esteroles de las células eucariotas y se cree que tienen una función de barrera en la membrana de ciertas bacterias ${ }^{62}$. Se ha relacionado el déficit de hopanoides con el aumento de la susceptibilidad a polimixinas y, por lo mismo, se cree que estas moléculas contribuyen a la insusceptibilidad de, por ejemplo, 
Burkholderia multivorans a esta clase de antimicrobianos $^{63}$. Otro mecanismo descrito en Burkholderia cenocepacia que le confiere resistencia natural a polimixinas es la presencia de proteasas en el espacio periplásmico ${ }^{64}$.

Por otra parte, hay evidencia de la importante frecuencia de resistencia a colistín en aislados de K. pneumoniae resistentes a carbapenémicos, con frecuencias que varían entre 9,7 y $32 \%$; con frecuencias intermedias de resistencia en $A$. baumannii, alrededor de 5\%, y más baja para $E$. coli y $P$. aeruginosa, $<1 \%{ }^{49}$.

Hasta este punto, todos los mecanismos descritos son mediados en el cromosoma. Lamentablemente Liu y cols ${ }^{65}$ describen en China el primer mecanismo plasmídico de resistencia a colistín en Enterobacteriaceae, tanto en animales como en humanos, denominado MCR-1, que corresponde a una fosfoetanolamina transferasa, capaz de modificar el sitio blanco y así disminuir la afinidad del colistín por el lípido A (la atracción electrostática). La trascendencia de este hallazgo es máxima, ya que nos adelanta en la era post-antibiótica, por las crecientes descripciones de BGN pandrogo-resistentes. El sobreuso de colistín en agricultura, tal como ocurre en Europa y Asia es una instancia crucial de selección de cepas resistentes, por lo que se hace extremadamente necesario que se tomen medidas gubernamentales para controlar este tipo de prácticas.

\section{Toxicidad}

En esta revisión se ha mencionado reiteradamente que debido a los efectos adversos estas moléculas fueron retiradas del mercado en la década de 1980 y que la razón de su re-introducción no se debió a que haya mejorado su perfil de seguridad, sino que fue una necesidad ante la inexistencia de alternativas terapéuticas frente a BGN XDR.

La nefrotoxicidad es la principal y más preocupante reacción adversa descrita para colistín. Lamentablemente los estudios que buscaron analizar la incidencia de toxicidad renal para esta polimixina, carecen de uniformidad en las definiciones de falla renal, y esto mismo explica que se describan valores que fluctúan, en general, entre $10-50 \%{ }^{66-77}$. No obstante lo anterior, es probable que actualmente la frecuencia de nefrotoxicidad sea más baja, ya que contamos con mejores unidades de pacientes críticos y preparados farmacéuticos más puros ${ }^{78,79}$. De hecho, en pacientes con fibrosis quística, CMS ha sido comparado favorablemente con los aminoglucósidos ${ }^{80}$. La dosis utilizada se ha relacionado en forma directamente proporcional con la incidencia de este evento adverso. Para algunos autores se asocia con la dosis total acumulada ${ }^{72,73}$ y para otros con la dosis diaria administrada ${ }^{74,75,81}$. Se ha descrito para pacientes que reciben CMS por más de dos semanas 3,7 veces más riesgo de nefrotoxicidad ${ }^{73} \mathrm{y}$, por esta razón, se debe evitar cursos prolongados de tratamiento con polimixinas cuando el contexto clínico lo permita. En la gran mayoría de los casos la falla renal aparece en los primeros siete días de tratamiento y la función renal se recupera tras la suspensión de éste, en al menos $90 \%$ de los $\operatorname{casos}^{10}$. El mecanismo específico de la nefropatía inducida por colistín no se conoce completamente, aunque modelos animales recientes sugieren que en su patogenia estarían involucrados el estrés oxidativo y la activación de algunas vías apoptóticas, como las caspasas ${ }^{82}$.

La neurotoxicidad es otra de las preocupaciones de los clínicos al usar polimixina $\mathrm{E}$, aunque su incidencia es mucho menor que el daño renal, es leve y además resuelve rápidamente tras la discontinuación del antimicrobiano ${ }^{79}$. Las parestesias son su principal manifestación y se estima su incidencia en una cifra cercana a $30 \%$ de los casos. Sin embargo, una gran lista de eventos adversos neurológicos han sido descritos con el uso de CMS, entre ellas: vértigo, debilidad muscular, hipoacusia, alteraciones visuales, confusión, alucinaciones, convulsiones, ataxia y bloqueo neuromuscular ${ }^{10}$.

\section{Terapia combinada}

El uso de terapias combinadas con CMS/colistín se basa en los siguientes elementos: con los esquemas de dosificación más agresivos, es decir, aquellos que recomiendan utilizar dosis de carga y terapias con dosificaciones de alrededor de 9.000.000 UI al día en pacientes adultos no obesos, se logran concentraciones plasmáticas máximas cercanas a $2 \mathrm{mg} / \mathrm{L}$, que corresponde al punto de corte de susceptibilidad para esta molécula. Por lo anterior, el uso de monoterapia, al menos teóricamente, es riesgosa y facilitaría la exposición al antimicrobiano en concentraciones incluso sub-inhibitorias, favoreciendo así los fenómenos de resistencia; más aún, in vitro se ha documentado que con concentraciones plasmáticas de 2,5 mg/L de CMS/colistín, sólo se produce un efecto bacteriostático sobre aislados con CIM entre 0,5-2,0 $\mathrm{mg} / \mathrm{L}$. Otras consideraciones que avalan la necesidad de terapia combinada son la descripción de heteroresistencia a colistín en $\mathrm{BGN}$ XDR, la presencia de recrecimiento en los ensayos de curva de muerte y la presencia, dependiente del aislamiento estudiado, de un notable efecto sinérgico ${ }^{29}$.

La sinergia, utilizando la técnica de cinética de muerte, es definida como la disminución de al menos dos $\log _{10}$ con la terapia combinada en comparación con el agente más activo a las $24 \mathrm{~h}$ de incubación o una concentración inhibitoria fraccionada (sigla en inglés FIC) $<0,5^{83}$. En general, la experiencia in vitro al ensayar CMS/colistín asociado a carbapenémicos contra distintos BGN, uti- 
lizando ensayos de cinética de muerte, ha demostrado resultados favorables particularmente sobre aislados de A. baumannii, destacando además una baja frecuencia de antagonismo y desarrollo de resistencia ${ }^{84,85}$. Estos resultados han motivado la utilización clínica de este tipo de combinaciones. Sin embargo, los resultados en el área clínica han sido menos auspiciosos y a veces contradictorios $^{86-88}$.

Particular importancia tiene el ensayo clínico prospectivo de Durante-Mangoni y cols ${ }^{89}$ quienes compararon el uso de colistín (control) como monoterapia versus colistín más rifampicina (rama experimental) en infecciones producidas por A. baumannii XDR, especialmente neumonía asociada a ventilación mecánica, enrolando 210 pacientes críticos de cinco centros hospitalarios italianos. La mortalidad a 30 días fue muy similar en ambas ramas, alrededor de $43 \%$, sin diferencia significativa desde el punto de vista estadístico. A pesar que la erradicación microbiológica de $A$. baumannii fue superior en la rama experimental (63 vs 47\%, respectivamente, $\mathrm{p}=0,034$ ), no hubo diferencias tampoco en la duración de la estancia hospitalaria. Debe destacarse que este estudio se inició el año 2008, de modo que hay varios aspectos a considerar según el conocimiento actual de PK/PD de colistín/CMS: no utilización de dosis de carga y subdosificación de colistín, además hubo uso de otros agentes antibacterianos en ambos grupos, incluso hasta en $70 \%$ del grupo control $(16 \% \text { de uso de meropenem })^{86,90}$. Aydemir y cols..$^{91}$, tampoco encontraron diferencias significativas en mortalidad en pacientes con neumonía asociada a ventilación mecánica por A. baumannii al comparar colistín vs colistín más rifampicina. La conclusión sistemática de todos los estudios, tanto pre-clínicos como clínicos, es la necesidad de más estudios multicéntricos, controlados y aleatorizados bien diseñados. Así, actualmente hay dos grandes estudios en desarrollo, uno en Europa, NCT01732250, en que se compara colistín dosis de carga 9.000.000 UI y luego 4,5 millones cada 12 h IV versus colistín dosis de carga 9.000.000 UI y luego 4,5 millones cada $12 \mathrm{~h}$ IV más meropenem $2 \mathrm{~g}$ cada $8 \mathrm{~h}$ IV (pacientes con función renal normal) en centros de Israel, Grecia e Italia y otro el NCT01597973 en varios centros de E.U.A., además de hospitales en Israel, Taiwán y Tailandia donde también se compara en una rama colistín, de acuerdo al conocimiento actual $\mathrm{PK} / \mathrm{PD}$, versus colistín más imipenem o meropenem $^{86}$.

\section{Conclusión}

En la actualidad contamos cada vez con menos opciones terapéuticas para combatir infecciones por bacterias gramnegativas extensamente resistentes a antimicrobianos. En este contexto, fue necesario re-introducir el colistín a la práctica clínica, molécula que había sido retirada previamente por toxicidad. Esta polimixina corresponde a un antimicrobiano lipopeptídico cíclico, con una interesante relación estructura-actividad, en la cual la positividad de sus cargas, aminas presentes en los residuos de ácido di-amino-butírico, le permiten una atracción electrostática con los fosfatos cargados negativamente del lípido A, favoreciendo una des-estructuración de la membrana externa y luego la inserción de la cola hidrofóbica (de naturaleza lipídica), produce una fusión entre la cara interna de la membrana externa y la cara que mira al espacio periplásmico de la membrana citoplasmática, induciendo -vía mecanismo(s) no totalmente dilucidado(s)- la muerte bacteriana.

A pesar que la farmacología moderna ha contribuido a aclarar algunas dudas sobre la administración de este agente antibacteriano, aún queda mucho por conocer. $\mathrm{Si}$ bien la resistencia global a este antimicrobiano es baja, a medida que ha aumentado su uso clínico se ha incrementado la descripción de resistencia en diferentes bacterias. Conceptualmente el mecanismo más importante es la modificación del LPS.

Por otra parte, el principal efecto adverso de este antimicrobiano es la toxicidad renal, seguido de la neurotoxicidad. Existen estudios de terapia combinada in vitro que han revelado resultados alentadores; sin embargo, a la fecha no hay evidencia clínica de que la asociación de colistín más un segundo agente logre impactar positivamente en la mortalidad.

\section{Resumen}

El resurgimiento de colistín para el tratamiento de bacilos gramnegativos extensamente resistentes a antimicrobianos a fines del siglo pasado es una de las características más importantes de la era post-antimicrobiana. $\mathrm{Su}$ espectro es reducido y cubre microorganismos con importancia clínica como Acinetobacter baumannii, Pseudomonas aeruginosa y Klebsiella pneumoniae. En contraste a lo que se vio en el pasado, la toxicidad descrita en la actualidad es menor, en parte explicado por las mejores preparaciones farmacéuticas y la optimización del manejo del paciente crítico. Mucha confusión se ha generado respecto a la dosificación del fármaco, debido a la distinta denominación, etiquetado y sugerencias de los laboratorios, a pesar de que el compuesto es el mismo. Por lo anterior, el llamado de los expertos es a utilizar un lenguaje común para referirnos a esta polimixina. Los estudios modernos de PK/PD han contribuido con nuevas formas de administrar y calcular las dosis de este antimicrobiano; no obstante, falta mucho por desarrollar en esta área que se posiciona como su gran debilidad. A pesar que la terapia combinada se sustenta sobre una base teórica lógica, no se ha demostrado que la asociación de colistín con un segundo agente logre disminuir la mortalidad. 


\section{Referencias bibliográficas}

1.- Magiorakos A P, Srinivasan A, Carey R B, Carmeli Y, Falagas M E, Giske C G, et al. Multidrug-resistant, extensively drug-resistant and pan drug resistant bacteria: an international expert proposal for interim standard definitions for acquired resistance. Clin Microbiol Infect 2012; 18: 268-81

2.- Appelbaum P C. 2012 and beyond: potential for the start of a second pre-antibiotic era? J Antimicrob Chemother 2012; 67: 2062-8.

3.- Arias C, Murray B. Antibiotic-resistant bugs in the 21 st Century-a clinical super challenge. N Engl J Med 2009; 360: 439-43.

4.- Cohen M L. Epidemiology of drug resistance: implications for a post-antimicrobial era. Science 1992; 257: 1050-55.

5.- Mella S, Riedel G, Twele L, Domínguez M, Bello H, González G. La conexión neumocócica. Rev Chilena Infectol 2013; 30 (1): 74-9.

6.- Payne D J, Gwynn M N, Holmes D J, Pompliano D L. Drugs for bad bugs: confronting the challenges of antibacterial discovery. Nat. Rev. Drug Discov 2007; 6 (1): 29-40.

7.- Infectious Diseases Society of America. The $10 \times$ '20 initiative: pursuing a global commitment to develop 10 new antibacterial drugs by 2020. Clin Infect Dis 2010; 50 (8): 1081-3.

8.- Boucher H W, Talbot G H, Bradley J S, Edwards J E, Gilbert D, Rice L B, et al. Bad bugs, no drugs: No ESKAPE! An update from the Infectious Diseases Society of America. Clin Infect Dis 2009; 48: 1-12.

9.- Fica A, Céspedes I, Gompertz M, Jalón M, Sakurada A, Sáez E. Colistín en infecciones nosocomiales por bacilos gramnegativos panresistentes. Rev Chilena Infectol 2007; 24 (5): 360-7

10.- Yahav D, Farbman L, Leibovici L, Paul M. Colistin: new lessons on an old antibiotic. Clin Microbiol Infect 2012; 18: 18-29.

11.- Li J, Nation R L, Turnidge J D, Milne R W, Coulthard K, Rayner C R, et al. Colistin: the reemerging antibiotic for multidrug-resistance gram negative bacterial infections. Lancet Infect Dis 2006; 6: 589-601.

12.- Cifuentes M, Silva F, García P, Bello H, Briceño, Calvo-A M, et al. Susceptibilidad antimicrobiana en Chile 2012. Rev Chilena Infectol 2014; 31 (2): 123-30.

13.- Cifuentes M, García P, San Martín P, Silva F, Zúñiga $\mathrm{J}$, Reyes $\mathrm{S}$, et al. Primer caso de detección de blaKPC en Chile: desde Italia a un hospital público de Santiago. Rev Chilena Infectol 2012; 29 (2): 224-8.

14.- Salles M J, Zurita J, Mejía C, Villegas M V. Resistant Gram-negative infections in the outpatient setting in Latin America. Epidemiol
Infect 2013; 141 (12): 2459-72.

15.- Bryskier A. Peptide antibiotics. Bryskier A $\left(1^{\circ}\right.$ ed). Antimicrobial Agents. Antibacterial and antifungals. ASM Press; Washington DC: 826-79.

16.- Bryskier A. Antibiotics and antibacterial agents: classifications and structure-activity relationship. Bryskier A (1ed). Antimicrobial Agents. Antibacterial and antifungals.ASM Press; Washington DC: 13-38.

17.- Vlekov T, Roberts K D, Nation R L, Thompson P E, Li J. Pharmacology of polymyxins: new insights into an "old" class of antibiotics. Future Microbiol 2013; 8: 711-24.

18.- Bergen P J, Landersdorfer C B, Zhang J, Zhao M, Lee H J, Nation R L, et al. Pharmacokinetics and pharmacodynamics of 'old' polymyxins: what is new? Diagn Microbiol Infect Dis 2012; 74: 213-23.

19.- Mella S, Sepúlveda M, González G, Bello H, Domínguez M. Aminoglucósidosaminociclitoles: Características estructurales y nuevos aspectos sobre su resistencia. Rev Chilena Infectol 2004; 21: 330-8.

20.- Zhang G, Meredith T C, Kahne D. On the essentiality of lipopolysaccharide to Gram negative bacteria. Curr Opin Microbiol 2013; 16 (6): 779-85.

21.- Holst $O$. The structures of core regions from enterobacterial lipopolysaccharides - an update. FEMS Microbiol Lett 2007; 271: 3-11.

22.- Nikaido H. Molecular basis of bacterial outer membrane permeability revisited. Microbiol Mol Biol Rev 2003; 67 (4): 593-656.

23.- Hancock R E. The bacterial outer membrane as a drug barrier. Trends Microbiol 1997; 5 (1): $37-42$.

24.- Hancock R E, Chapple D S. Peptide antibiotics. Antimicrob Agents Chemother 1999; 43 (6): 1317- 23.

25.- Falagas M E, Kasiakou S K. Colistin: The revival of polymyxins for the management of multidrug-resistant gram-negative bacterial infections. Clin Infect Dis 2005; 40: 1333-41.

26.- Walkty A, DeCorby M, Nichol K, Karlowsky J A, Hoban D J, Zhanel G G. In vitro activity of colistin (polymyxin E) against 3,480 isolates of gram-negative bacilli obtained from patients in Canadian hospitals in the CANWARD study, 2007-2008. Antimicrob Agents Chemother 2009; 53: 4924-6.

27.- Clinical and Laboratory Standards Institute. Performance Standards for Antimicrobial Susceptibility Testing: Twenty-fifth Informational Supplement M100-S25. In: CLSI, Wayne, PA, USA, 2015

28.- European Committee on Antimicrobial Susceptibility Testing. Clinical breakpointsbacteria (v 6.0). Available at: http://www.eucast. org/clinical_breakpoints/. Accessed 9 March 2016.
29.- Ortwine J K, Kaye K S, Li J, Pogue J. Colistin: understanding and applying recent pharmacokinetic advances. Pharmacotherapy 2015; 35 (1): 11-6.

30.- Forest Laboratories UK Limited. Colomycin [packageinsert]. Dartford, Kent, U.K.; 2012.

31.- Monarch Pharmaceuticals, Inc. Coly-Mycin M Parenteral [package insert]. Bristol, TN; 2006.

32.- Nation R L, Li J, Cars O, Couet W, Dudley M N, Kaye K S, et al. Consistent global approach on reporting of colistin doses to promote safe and effective use. Clin Infect Dis 2014; 58: 139-41.

33.- Nation R L, Li J, Turnidge J D. The urgent need for clear and accurate information on the polymyxins. Clin Infect Dis 2013; 57 (11): 1656-7.

34.- Theuretzbacher U. Product information for parenteral colistin varies substantially across Europe. J Antimicrob Chemother 2014; 69: 1987-92.

35.- Kassamali Z, Rotschafer J C, Jones R N, Prince R A, Danziger L H. Polymyxins: wisdom does not always come with age. Clin Infect Dis 2013; 57 (6): 877-83.

36.- Falagas M E, Kasiakou S K. Use of international units when dosing colistin will help decrease confusion related to various formulations of the drug around the world. Antimicrob Agents Chemother 2006; 50 (6): 2274-5.

37.- Bergen P J, Li J, Rayner C R, Nation R L. Colistin methanesulfonate is an inactive prodrug of colistin against Pseudomonas aeruginosa. Antimicrob Agents Chemother 2006; 50: 1953-8.

38.- Li J, Milne R W, Nation R L, Turnidge J D, Coulthard K, Valentine J. Simple method for assaying colistin methanesulfonate in plasma and urine using high- performance liquid chromatography. Antimicrob Agents Chemother 2002; 46: 3304-7.

39.- Plachouras D, Karvanen M, Friberg L E, Papadomichelakis E, Antoniadou A, Tsangaris I, et al. Population pharmacokinetic analysis of colistin methanesulfonate and colistin after intravenous administration in critically ill patients with infections caused by Gram-negative bacteria. Antimicrob Agents Chemother 2009; 53: 3430-6.

40.- Garonzik S M, Li J, Thamlikitkul V, Paterson D L, Shoham S, Jacob J, et al. Population pharmacokinetic of colistin methanesulfonate and formed colistin in critically ill patients from a multicenter study to provide dosing suggestions for various categories of patients. Antimicrob Agents Chemother 2011; 55: 3284-94.

41.- Wallace S J, Li J, Nation R L, Rayner C R, Taylor D, Middleton D, et al. Subacute toxicity of colistin methanesulfonate in rats: comparison of various intravenous dosage regimens. 
Antimicrob Agents Chemother 2008; 52: 1159-61.

42.- Bergen P J, Li J, Nation R L, Turnidge J D, Coulthard K, Milne R W. Comparison of once-, twice- and thrice-daily dosing of colistin on antibacterial effect and emergence of resistance: studies with Pseudomonas aeruginosa in an in vitro pharmacodynamic model. J Antimicrob Chemother 2008; 61: 636-42.

43.- Dudhani R V, Turnidge J D, Coulthard K, Milne R W, Rayner C R, Li J, et al. Elucidation of the pharmacokinetic/pharmacodynamic determinant of colistin activity against Pseudomonas aeruginosa in murine thigh and lung infection models. Antimicrob. Agents Chemother 2010; 54 (3): 1117-24.

44.- Dudhani R V, Turnidge J D, Nation R L, $\mathrm{Li} \mathrm{J}$. fAUC/MIC is the most predictive pharmacokinetic/ pharmacodynamic index of colistin against Acinetobacter baumannii in murine thigh and lung infection models. J Antimicrob Chemother 2010; 65 (9): 1984-90.

45.- Markantonis S L, Markou N, Fousteri M, Sakellaridis N, Karatzas S, Alamanos I, et al. Penetration of colistin into cerebrospinal fluid. Antimicrob Agents Chemother 2009; 53: 4907- 10.

46.- Poudyal A, Howden B P, Bell J M, Gao W, Owen R J, Turnidge J D, et al. In vitro pharmacodynamics of colistin against multidrug- resistant Klebsiella pneumoniae. J Antimicrob Chemother 2008; 62 (6): 1311-8.

47.- Owen R J, Li J, Nation R L, Spelman D. In vitro pharmacodynamics of colistin against Acinetobacter baumannii clinical isolates. J Antimicrob Chemother 2007; 59: 473-7.

48.- Antoniadou A, Kontopidou F, Poulakou G, Koratzanis E, Galani I, Papadomichelakis E, et al. Colistin-resistant isolates of Klebsiella pneumoniae emerging in intensive care unit patients: first report of a multiclonal cluster. J Antimicrob Chemother 2007; 59: 786-90.

49.- Giske C G. Contemporary resistance trends and mechanisms for colistin, temocillin, fosfomycin, mecillinam and nitrofurantoin. Clin Microbiol Infect (2015), doi:10.1016/j.cmi.2015.05.022.

50.- Adams M D, Nickel G C, Bajaksouzian S, Lavender H, Murthy A R, Jacobs M R, et al. Resistance to colistin in Acinetobacter baumannii associated with mutations in the PmrAB two-component system. Antimicrob Agents Chemother 2009; 53: 3628 -34.

51.- Blair J M, Webber M A, Baylay A J, Ogbolu D O, Piddock L J. Molecular mechanisms of antibiotic resistance. Nat Rev Microbiol 2015; 13: 42-51.

52.- Beceiro A, Llobet E, Aranda J, Bengoechea J A, Doumith M, Hornsey M, et al. Phosphoethanolamine modification of lipid A in colistin-resistant variants of Acinetobacter baumannii mediated by the pmrAB two-component regulatory system. Antimicrob Agents Chemother 2011; 55: 3370-9.

53.- Llobet E, Campos M A, Gimenez P, Moranta D, Bengoechea J A. Analysis of the networks controlling the antimicrobial-peptide dependent induction of Klebsiella pneumoniae virulence factors. Infect Immun 2011; 79 (9): 3718-32.

54.- Moffatt J H, Harper M, Harrison P, Hale J D, Vinogradov E, Seemann T, et al. Colistin resistance in Acinetobacter baumannii is mediated by complete loss of lipopolysaccharide production. Antimicrob Agents Chemother 2010; 54 (12): 4971-7.

55.- Moffatt J H, Harper M, Adler B, Nation R L, Li J, Boyce J D. The insertion sequence ISAball is involved in colistin resistance and loss of lipopolysaccharide in Acinetobacter baumannii. Antimicrob Agents Chemother 2011; 55: 3022-4.

56.- Henry R, Vithanage N, Harrison P, Seemann T, Coutts S, Moffatt J H, et al. Colistin-resistant, lipopolysaccharide-deficient Acinetobacter baumannii responds to lipopolysaccharide loss through increased expression of genes involved in the synthesis and transport of lipoproteins, phospholipids, and poly- $\beta-1,6-$ $\mathrm{N}$-acetylglucosamine. Antimicrob Agents Chemother 2012; 56: 59-69.

57.- Llobet E, Tomas J M, Bengoechea J A. Capsule polysaccharide is a bacterial decoy for antimicrobial peptides. Microbiology 2008; 154 (12): 3877-86.

58.- Pamp S J, Gjermansen M, Johansen H K, Tolker-Nielsen T. Tolerance to the antimicrobial peptide colistin in Pseudomonas aeruginosa biofilms is linked to metabolically active cells, and depends on the pmr and mexAB-oprM genes. Mol Microbiol 2008; 68 (1): 223-40.

59.- Padilla E, Llobet E, Domenech-Sánchez A, Martínez-Martínez L, Bengoechea J A, Alberti S. Klebsiella pneumoniae AcrAB efflux pump contributes to antimicrobial resistance and virulence. Antimicrob Agents Chemother 2010; 54 (1): 177-83.

60.- Warner D M, Levy S B. Different effects of transcriptional regulators MarA, SoxS and Rob on susceptibility of Escherichia coli to cationic antimicrobial peptides (CAMPs): Rob-dependent CAMP induction of the marRAB operon. Microbiology 2010; 156 (Pt 2): 570-8.

61.- Fehlner-Gardiner C C, Valvano M A. Cloning and characterization of the Burkholderia vietnamiensis nor $\mathrm{M}$ gene encoding a multidrug efflux protein. FEMS Microbiol Lett 2002; 215 (2): 279-83.

62.- Schmerk C L, Bernards M A, Valvano M A. Hopanoid production is required for low-pH tolerance, antimicrobial resistance, and motility in Burkholderia cenocepacia. J Bacteriol 2011; 193 (23): 6712-23.

63.- Malott R J, Steen-Kinnaird B R, Lee T D, Speert D P. Identification of hopanoid biosynthesis genes involved in polymyxin resistance in Burkholderia multivorans. Antimicrob Agents Chemother 2012; 56 (1): 464-71.

64.- Loutet S A, Valvano M A. Extreme antimicrobial peptide and polymyxin B resistance in the genus Burkholderia. Front Cell Infect Microbiol 2011; 1: 6.

65.- Liu Y-Y, Wang Y, Walsh T R, Yi L-X, Zhang R, Spencer J, et al. Emergence of plasmid-mediated colistin resistance mechanism MCR-1 in animals and human beings in China: a microbiological and molecular biological study. Lancet Infect Dis 2015; Published Online November 18, 2015 http://dx.doi.org/10.1016/ S1473-3099(15)00424-7.

66.- Falagas M E, Rafailidis P I, Ioannidou E, Alexiou V G, Matthaiou D K, Karageorgopoulos $\mathrm{D} E$, et al. Colistin therapy for microbiologically documented multidrug resistant Gram-negative bacterial infections: a retrospective cohort study of 258 patients. Int J Antimicrob Agents 2010; 35: 194-9.

67.- Doshi N M, Mount K L, Murphy C V. Nephrotoxicity associated with intravenous colistin in critically ill patients. Pharmacotherapy 2011; 31: 1257-64.

68.- Gauthier T P, Wolowich W R, Reddy A, Cano E, Abbo L, Smith L B. Incidence and predictors of nephrotoxicity associated with intravenous colistin in overweight and obese patients. Antimicrob Agents Chemother 2012; 56: 2392-6.

69.- Falagas M E, Rizos M, Bliziotis I A, Rellos K, Kasiakou S K, Michalopoulos A. Toxicity after prolonged (more than four weeks) administration of intravenous colistin. BMC Infect Dis 2005; 5: 1 .

70.- Falagas M E, Kasiakou S K, Kofteridis D P, Roditakis G, Samonis G. Effectiveness and nephrotoxicity of intravenous colistin for treatment of patients with infections due to polymyxin-only-susceptible (POS) gramnegative bacteria. Eur J Clin Microbiol Infect Dis 2006; 25: 596-9.

71.- Cheng C Y, Sheng W H, Wang J T, Chen Y C, Chang S C. Safety and efficacy of intravenous colistin (colistin methanesulphonate) for severe multidrug-resistant gram-negative bacterial infections. Int J Antimicrob Agents 2010; 35: 297-300.

72.- Falagas M E, Fragoulis K N, Kasiakou S K, Sermaidis G J, Michalopoulos A. Nephrotoxicity of intravenous colistin: a prospective evaluation. Int J Antimicrob Agents 2005; 26: 504-7.

73.- Hartzell J D, Neff R, Ake J, Howard R, Olson S, 
Paolino K, et al. Nephrotoxicity associated with intravenous colistin (colistimethate sodium) treatment at a tertiary care medical center. Clin Infect Dis 2009; 48: 1724-8.

74.- Deryke C A, Crawford A J, Uddin N, Wallace M R. Colistin dosing and nephrotoxicity in a large community teaching hospital. Antimicrob Agents Chemother 2010; 54: 4503-5.

75.- Pogue J M, Lee J, Marchaim D, Yee V, Zhao J J, Chopra T, et al. Incidence of and risk factors for colistin-associated nephrotoxicity in a large academic health system. Clin Infect Dis 2011; 53: 879-84.

76.- Kim J, Lee K H, Yoo S, Pai H. Clinical characteristics and risk factors of colistininduced nephrotoxicity. Int J Antimicrob Agents 2009; 34: 434-8.

77.- Ko H, Jeon M, Choo E, Lee E J, Kim Th, Jun J B, et al. Early acute kidney injury is a risk factor that predicts mortality in patients treated with colistin. Nephron Clin Pract 2011; 117: c284-8.

78.- Falagas M E, Rafailidis P I. Nephrotoxicity of colistin: new insight into an old antibiotic. Clin Infect Dis 2009; 48: 1729-31.

79.- Falagas M E, Kasiakou S K. Toxicity of polymyxins: a systematic review of the evidence from old and recent studies. Crit Care 2006; 10: R27.

80.- Etherington C, Bosomworth M, Clifton I, Peckham D G, Conway S P. Measurement of urinary N-acetyl- $\beta$-D- glucosaminidase in adult patients with cystic fibrosis: Before, during and after treatment with intravenous antibiotics. J Cyst Fibros 2007; 6: 67-73.

81.- Rattanaumpawan $P$, Ungprasert $P$, Thamlikitkul V. Risk factors for colistinassociated nephrotoxicity. J Infect 2011; 62: 187-90.

82.- Ozkan G, Ulusoy S, Orem A, Alkanat M, Mungan S, Yulug E, et al. How does colistininduced nephropathy develop and can it be treated?. Antimicrob Agents Chemother 2013; 57 (8): 3463-9.

83.- Lorian V, editor: Antibiotics in laboratory medicine, Philadelphia, 2005, Lippincott Williams and Wilkins.

84.- Zusman O, Avni T, Leibovici L, Adler A, Friberg L, Stergiopoulou T, et al. Systematic review and meta-analysis of in vitro synergy of polymyxins and carbapenems. Antimicrob Agents Chemother 2013; 57: 5104-11.

85.- Tripodi M F, Durante-Mangoni E, Fortunato R, Utili R, Zarrilli R. Comparative activities of colistin, rifampicin, imipenem and sulbactam/ampicillin alone or in combination against epidemic multidrug-resistant Acinetobacter baumannii isolates producing OXA-58 carbapenemases. Int J Antimicrob Agents 2007; 30: 537-40.

86.- Nation R L, Li J, Cars O, Couet W, Dudley M N, Kaye K S, et al. Framework for optimization of the clinical use of colistin and polymyxin B: the Prato polymyxin consensus. Lancet Infect Dis 2015; 15: 225-34.

87.- Poulikakos P, Tansarli G S, Falagas M E. Combination antibiotic treatment versus monotherapy for multidrug-resistant, extensively drug-resistant and pandrugresistant Acinetobacter infections: a systematic review. Eur J Clin Microbiol Infect Dis 2014; 33: 1675-85.

88.- Paul M, Carmeli Y, Durante-Mangoni E, Mouton J W, Tacconelli E, Theuretzbacher U, et al. Combination therapy for carbapenemresistant Gram-negative bacteria. J Antimicrob Chemother 2014; 69: 2305-9.

89.- Durante-Mangoni E, Signoriello G, Andini R, Mattei A, De Cristoforo M, Murino P, et al. Colistin and rifampicin compared with colistin alone for the treatment of serious infections due to extensively drug-resistant Acinetobacter baumannii: a multicenter, randomized clinical trial. Clin Infect Dis 2013; 57 (3): 349-58.

90.- Gauthier T P. Rifampicin plus colistin in the era of extensively drug-resistant Acinetobacter baumannii infections. Clin Infect Dis 2013; 57 359-61.

91.- Aydemir H, Akduman D, Piskin N, Comert F, Horuz E, Terzi, A, et al. Colistin vs the combination of colistin and rifampicin for the treatment of carbapenem-resistant Acinetobacter baumannii ventilator-associated pneumonia. Epidemiol Infect 2013; 141: 1214-22. 